\title{
Gender and collaboration patterns in a temporal scientific authorship network
}

${\text { Gecia Bravo-Hermsdorff }{ }^{*} \text { (D), Valkyrie Felso }}^{1,2}$, Emily Ray ${ }^{3,4}$, Lee M. Gunderson ${ }^{5}$, Mary E. Helander ${ }^{6}$, Joana Maria ${ }^{4}$ and Yael Niv ${ }^{1,2}$

\section{*Correspondence:}

gecia.bravo@gmail.com

'Princeton Neuroscience Institute,

Princeton University, Washington $\mathrm{Rd}$, 08544, Princeton, NJ, USA

Full list of author information is available at the end of the article

\begin{abstract}
One can point to a variety of historical milestones for gender equality in STEM (science, technology, engineering, and mathematics), however, practical effects are incremental and ongoing. It is important to quantify gender differences in subdomains of scientific work in order to detect potential biases and monitor progress. In this work, we study the relevance of gender in scientific collaboration patterns in the Institute for Operations Research and the Management Sciences (INFORMS), a professional society with sixteen peer-reviewed journals. Using their publication data from 1952 to 2016, we constructed a large temporal bipartite network between authors and publications, and augmented the author nodes with gender labels. We characterized differences in several basic statistics of this network over time, highlighting how they have changed with respect to relevant historical events. We find a steady increase in participation by women (e.g., fraction of authorships by women and of new women authors) starting around 1980 . However, women still comprise less than $25 \%$ of the INFORMS society and an even smaller fraction of authors with many publications. Moreover, we describe a methodology for quantifying the structural role of an authorship with respect to the overall connectivity of the network, using it to measure subtle differences between authorships by women and by men. Specifically, as measures of structural importance of an authorship, we use effective resistance and contraction importance, two measures related to diffusion throughout a network. As a null model, we propose a degreepreserving temporal and geometric network model with emergent communities. Our results suggest the presence of systematic differences between the collaboration patterns of men and women that cannot be explained by only local statistics.

Keywords: Authorship network, Collaboration patterns, Temporal network, Gender in STEM
\end{abstract}

\section{Introduction}

Recent years have seen an increase in awareness and discussion of systematic gender biases in academia. A slew of studies and opinion publications (Raymond 2013; Schrouff et al. 2019) have highlighted often unintentional, but nevertheless pervasive, biases in integral aspects of academic careers, such as hiring practices (Moss-Racusin et al. 2012), funding decisions (Witteman et al. 2019), peer review (Helmer et al. 2017), and representation of women as speakers in conferences (Nittrouer et al. 2017). It is therefore important to precisely quantify gender differences in subdomains of academic work as a first step towards detecting potential biases and finding appropriate solutions.

One domain in which network science could offer insight is in the study of gender differences in authorship and collaboration patterns. Indeed, there have been several studies

(c) The Author(s). 2019 Open Access This article is distributed under the terms of the Creative Commons Attribution 4.0 International License (http://creativecommons.org/licenses/by/4.0/), which permits unrestricted use, distribution, and reproduction in any medium, provided you give appropriate credit to the original author(s) and the source, provide a link to the Creative Commons license, and indicate if changes were made. 
on this topic. For example, Araújo et al. (2017) analyzed a dataset containing more than 270,000 academics from a wide variety of fields (engineering, arts, biological, exact, and social sciences) in Brazil. They found that, across all fields, men were more likely to collaborate with other men, while for women, the gender ratio of their collaborations more closely matched that of the relevant academic population. In contrast, Karimi et al. (2018) showed that, in a research community in organizational science based in the US and Europe, women exhibited more gender homophily. Jadidi et al. (2018) investigated gender differences in a temporal network spanning 47 years with publication and citation data of over one million computer scientists, concluding that homophily has been increasing recently for both genders. Additionally, they found that women have a higher dropout rate than men, especially at the beginning of their careers. West et al. (2013) studied gender differences in a corpus containing over eight million publications in humanities, social and natural sciences. They found that, overall, gender differences in number of publications have been decreasing over time. However, in some fields, women remain disproportionately underrepresented as first, last, and solo authors.

While these studies provide a glimpse into gender differences in authorship and collaboration networks in academia, they tend to focus on local statistics (i.e., measures that depend only on neighboring connections, such as number of publications, homophily, and author order). In contrast, relatively less is known about the correlation between author gender and their roles in the global structure of the network. Indeed, appropriately characterizing these differences is a nontrivial task, as local and global measures are often intertwined.

In this work, we investigate gender differences over time using both local and global measures in a large corpus of publications from 1952 to 2016 in journals affiliated with the Institute for Operations Research and the Management Science (INFORMS) - the predominant professional society for the disciplines of operations research and management science (About INFORMS 2019). These fields are both squarely within STEM (science, technology, engineering, and mathematics), where the degree of gender imbalance has traditionally been considered more severe (but see Leslie et al. (2015)).

We represent the data as a temporal bipartite network (between authors and publications), and first characterize several local statistics of this network, highlighting their change with respect to potentially relevant historical events. We then describe a methodology for quantifying differences with respect to global structure (conditioned on several relevant local statistics), which could be of independent interest.

Specifically, we consider global structure to be related to the prototypical global process of diffusion. To this end, we selected two measures related to such dynamics, namely, effective resistance (Spielman and Srivastava 2011; Chandra et al. 1989; Christiano et al. 2011) and contraction importance (Bravo-Hermsdorff and Gunderson 2019), to measure the global importance of an author-publication connection. To account for local statistics, we describe a simple temporal and geometric null model, with only two free parameters controlling the emergence and size of communities. This null model explicitly replicates the observed yearly degree distributions for publications, as well as the genders and publication histories of each individual author. Importantly, the mechanism used to decide which authors participate in which publications is blind to the author gender. By comparing the null model to the observed network data, we can identify differences in the structural role played by men and women authorships beyond local statistics. Given 
the increased interest in the relatively new field of fairness in machine learning (Liu et al. 2018; Chouldechova and Roth 2018), the type of graph-theoretical analysis that we propose here could be useful for bias detection in other social networks.

\section{The dataset: a temporal, bipartite network between authors and their publications in INFORMS}

In this section, we introduce some relevant information about the INFORMS society, and describe our data acquisition and cleaning methods.

\section{The INFORMS society}

INFORMS was founded in 1995 with the merging of two societies: the Operations Research Society of America (ORSA) and The Institute of Management Sciences (TIMS) (Horner 2017), two societies that had already been linked prior to this time. For instance, they had many members in common (ORSA/TIMS collaboration 1973), had hosted joint meetings since 1961 (Hall 1983), and jointly sponsored two journals (Mathematics of Operations Research, founded in 1976, and Marketing Science, founded in 1982). INFORMS currently publishes 16 peer-reviewed journals (INFORMS Publications 2019), the oldest of which are the flagship journals of the two original societies: Operations Research, first published in 1952 by ORSA and Management Science, first published in 1954 by TIMS. As of December 2017, the society reports to have over 12,500 members (About INFORMS 2019), around 20\% of which identify as women (Denton 2017).

The INFORMS society has historically worked towards identifying and mitigating gender bias, making it particularly attractive for our study. For example, in 2006, then-president Mark S. Daskin founded a diversity committee "to assess whether or not there is any sort of problem with diversity within INFORMS” (Nagurney 2007). In 2017, the society created a "Diversity, Equity, and Inclusion" initiative, including the formation of a committee with

"[...] a broad charge that includes monitoring the diversity of our membership and seeking out, creating and maintaining best practices for INFORMS to improve diversity and inclusion-related performance." (Denton 2017)

\section{Data acquisition, data cleaning, and gender assignments}

We constructed a bipartite authorship network using the publications from 16 peerreviewed journals affiliated with INFORMS from 1952 to $2016 .{ }^{1}$ Publication records were acquired using INFORMS PubsOnline (in the form of BibTeX entries) and the CrossRef REST API (The Institute for Operations Research and the Management Sciences 2019; CrossRef REST API 2019). Nodes in the network are of two types: author or publication, and edges denote an authorship of a publication. Metadata include author name, author order, publication title, year, and journal.

We classified authors by gender using the commercial package Genderize.io API (Genderize.io 2019), which associates to each first name a gender (woman or man) and a confidence score ranging from 0 to 100 . We thresholded acceptable gender labels as

\footnotetext{
${ }^{1}$ The 16 journals in our dataset are not identical to the 16 journals currently published by INFORMS as one journal was removed during our period of interest (Management Technology merged with Management Science in 1965), and one was added after our period of interest (INFORMS introduced a new journal, INFORMS Journal on Optimization in 2018).
} 
those with a confidence level above 80 , noting that this could still yield misclassifications. Author nodes with confidence below 80 were initially marked as "unknown" gender.

We performed two data cleaning steps: (1) additional gender classification and (2) author node combination. For (1), all unknown-labeled authors with more than 6 publications were manually classified via internet searches. In addition, we verified the gender of the 100 most prolific woman-labeled authors, as well as the 100 most prolific men-labeled authors, and corrected the labels for any who had been misclassified. These misclassifications occurred mostly for given names that were historically used for men and shifted to women over time (e.g., Aubrey, Leslie, Sandy). For (2), we combined multiple nodes that referenced the same author (usually due to publication under a variety of names). For example, Robert Eugene Donald Woolsey, a prolific and well-known figure in the INFORMS society, published under the names "Eugene Woolsey", "Gene Woolsey", "R. E. D. Woolsey", "R. E. Woolsey”, and "Robert E. D. Woolsey”. We manually verified the 100 most prolific author nodes (along with any others noticed while examining the data) by searching for duplicate last (family) names and combining authors as needed.

The resulting network contains 23875 publication nodes, 22911 author nodes, 50527 edges, and 4587 connected components. Of the author nodes, 16179 are labeled as men, 2997 as women, and 3735 as unknown. The giant (i.e., largest) component of the network contains 13520 author nodes, 16604 publication nodes, and 38169 edges. Often, in studies of collaboration networks, one "projects" the network to a single type of node (e.g., by keeping the author nodes and replacing the publication nodes with cliques of edges between those authors) (Araújo et al. 2017; Karimi et al. 2018; Liu et al. 2005; Abbasi et al. 2012; Chen et al. 2017; Jadidi et al. 2018). However, we kept the original bipartite structure in all of our analysis, as projecting the network loses structural information (Kitsak 2017) (e.g, a publication with three authors would be identical to three publications between all pairs of those authors). We also kept the connections by authors of unknown gender to maintain the overall network connectivity.

\section{Local statistics: participation rates by women are increasing, but remain far from gender parity}

The broad strokes of gender asymmetry are easily seen with simple measures, such as participation rates. In this section, we quantify and discuss the evolution of several local statistics in the INFORMS authorship network (additional local statistics are provided in Additional file 2: Table S1).

\section{Relevant historical milestones}

While gender discrimination continues to be an issue in academic environments, actions have been taken to mitigate this discrepancy. To place the data in the relevant historical context, we highlight in our graphs the timing of two notable examples of such events: ${ }^{2}$

1. Title IX of the Education Amendments Act of 1972, which states:

No person in the United States shall, on the basis of sex, be excluded from participation in, be denied the benefits of, or be subjected to discrimination under any education program or activity receiving Federal financial assistance.

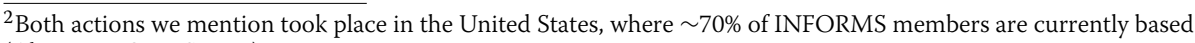
(About INFORMS 2019). 
While Title IX focused primarily on student athletics, the policy has had

far-reaching consequences. For example, the law has been credited with increasing access to college education for women (US Department of Justice 2012) and supporting applications to further gender equality for those employed in academia (Walters and McNeely 2010).

2. The Family and Medical Leave Act of 1993 (FMLA). This law protects employees who take leave from work for family or medical reasons. As women have traditionally performed most of the child care in the family, it represents another major milestone for women's rights in the United States, where family leave (including for childbirth) was not otherwise available or protected by law (Klerman et al. 2012).

\section{Participation rates by women and men over time}

The fractions of new women authors and of authorships by women have both been increasing since $\sim 1980$ (Fig. 1). However, both measures remain more than a factor of 2 from gender parity. In fact, a crude extrapolation of the fraction of new women authors (a weighted linear regression from 1976-2016) yields an estimate of gender parity by $2062 \pm 5$. The same extrapolation for the fraction of women authorships gives an estimate of gender parity by $2083 \pm 3$ (standard deviations were computed by propagating the errors due to the covariance of the slope and intercept of the linear fit).

The fraction of authorships by women is consistently lower than the fraction of new women authors, reflecting the fact that women continue to have a lower average number of publications than men (Fig. 2 and Additional file 1: Movie S1). Moreover, the cumulative degree distributions further suggest that women who have many publications are

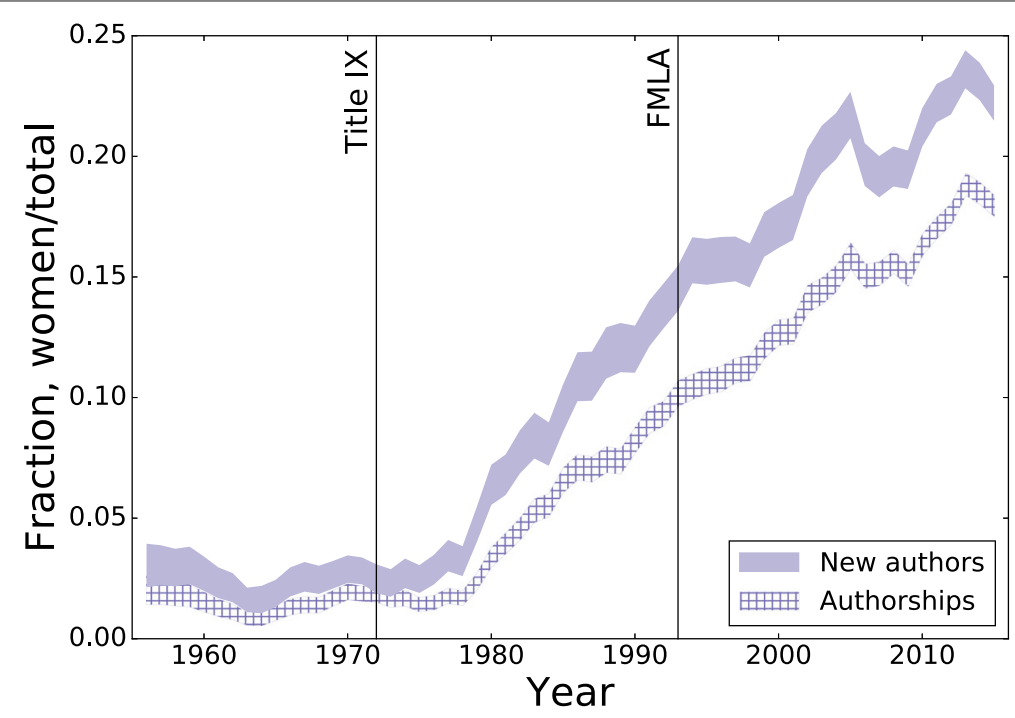

Fig. 1 Participation rate by women in INFORMS has been increasing since $\sim 1980$, but remains well below gender parity. Hatched: Fraction of authorships by women over time. Solid: Fraction of new women authors over time. The data were binned in a moving window centered around the plotted year. To allow for better statistics, for 1956-1963 the window widths were 8 years, for 1964-1971 they were 6 years, and the remainder were 4 years. Fractions were calculated excluding authors without a gender label. Shading denotes \pm 1 standard error of the mean, estimated treating the gender labels of all datapoints in the relevant window as a collection of i.i.d. Bernoulli random variables (i.e., $p \pm \sqrt{p(1-p) / n}$, where $p$ is the fraction and $n$ is the number of datapoints in the window). Note that due to the binning the rise in the curves in $\sim 1978$ reflects an increase of participation by women starting in $\sim 1980$ 

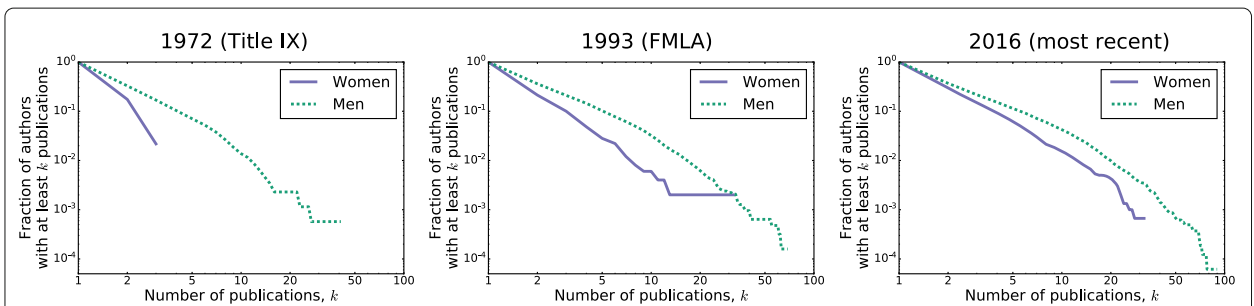

Fig. 2 Evolution of the cumulative degree distributions in the INFORMS network suggests that women are disproportionately underrepresented among authors with many publications. Plots display the normalized cumulative author degree distribution for the indicated year, i.e., the vertical axis is the fraction of authors of this gender with at least that number of publications. Since there are more men than women in the network, the degree distribution for men is expected to extend to more publications than that for women. However, the steeper slope of the distribution for women (in log scale) suggests their systematic underrepresentation, especially in the high-publication tail of the distribution (quantified in Fig. 3). See Additional file 1 for a video of the cumulative degree distributions over time

disproportionately rare. We quantified this effect by measuring the power-law exponent of the degree distributions, and found a significantly steeper slope for women (Fig. 3). This claim is further supported by the results shown in Fig. 4 .

\section{Two diffusion-based measures for quantifying}

\section{the importance of a connection to the global structure of the network}

We now discuss the measures we used to quantify the importance of a connection between an author and publication to the overall connectivity of the network (as opposed to local characteristics such as degree and other node/edge attributes). Our motivation for focusing on these particular measures is twofold: their use in graph algorithms relevant to the field of network science, and their direct relation with diffusion (arguably the simplest process that is sensitive to the global structure of a network). Additional global statistics of the INFORMS network are contained in Additional file 2: Table S1.
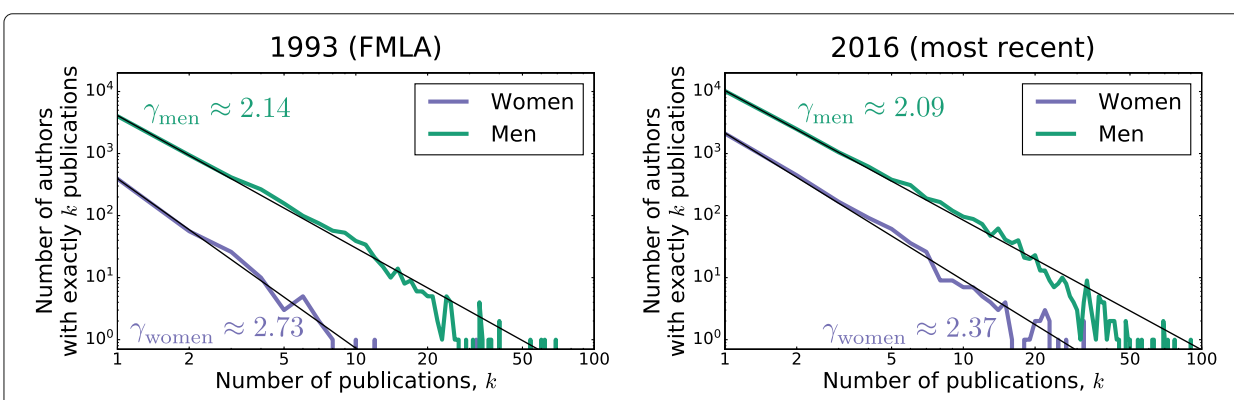

Fig. 3 Women are disproportionately underrepresented among authors with many publications in INFORMS. There appears to be an additional underrepresentation of women among authors with many publications, beyond what would already be expected by the lower proportion of women authors in the data. To quantify this effect, we modeled the degree distributions of each gender as a power law, using the exponent $\gamma$ as a measure of how "heavy" the tail of these distributions are. By considering the number of authors with $k$ publications as an independent Poisson variable with mean $n(k)=c k^{-\gamma}$, we maximized the likelihood of the data over the space of $c$ and $\gamma$. The results showed a steeper power law for women $\left(\gamma_{\text {women }} \approx 2.73\right.$ vs. $\gamma_{\text {men }} \approx 2.14$ in 1993 and $\gamma_{\text {women }} \approx 2.37$ vs. $\gamma_{\text {men }} \approx 2.09$ in 2016). To quantify the significance of this difference, we repeatedly randomized the gender labels of the nodes and considered the distributions of the fitted exponents $\gamma$ as the null distribution for the observed result. We found that the $z$-scores of the observed power-law exponents were about -9.4 for men and +11.6 for women in 1993, and about -6.9 for men and +10.5 for women in 2016, all highly significant. This indicates that women are disproportionately underrepresented among authors with many publications in INFORMS. However, this difference seems to be attenuating, as suggested by the decrease in the difference between $\gamma_{\text {women }}$ and $\gamma_{\text {men }}$ during this time 

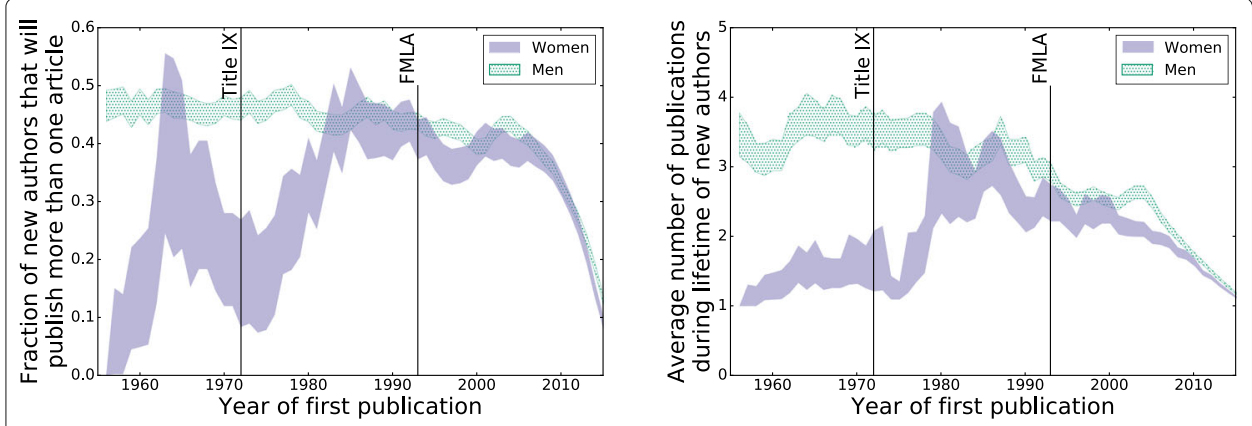

Fig. 4 In the later years, the fraction of new INFORMS women authors with more than one publication is similar to that of men, but their average number of publications remains lower. Left: Fraction of new authors that will publish again in INFORMS by 2016, as a function of their first year of publication. Right: Average total number of publications (as of 2016) by an author, also as a function of their first year of publication. A moving window was applied as in Fig. 1. Shading denotes \pm 1 standard error of the mean (computed as in Fig. 1 for the left plot, and using $\mu \pm \sigma / \sqrt{n}$, i.e., assuming a sum of independent Poisson variables, for the right plot). The decrease in both quantities in later years is simply due to the fact that recent new authors have had less time to publish again

The evolution of diffusion is governed by the graph Laplacian (defined as $L=D-A$, where $A$ is the adjacency matrix, and $D$ is the diagonal matrix of node degrees). Indeed, many questions about the connectivity of a network (e.g., max-cut/min-flow problems (Christiano et al. 2011), and community detection (Fiedler 1973)) have efficient solutions that rely on the graph Laplacian (Teng 2010). In this section, we describe two measures of edge importance derived from the action of the Laplacian (more specifically, its pseudoinverse).

\section{Effective resistance $\Omega$}

A measure known as effective resistance $\Omega$ naturally arises when quantifying the importance of an edge with respect to preserving the action of the graph Laplacian (Spielman and Srivastava 2011). The effective resistance $\Omega_{e}$ of an edge $e=\left(v_{1}, v_{2}\right)$ is defined as

$$
\Omega_{e}=b_{e}^{\top} L^{\dagger} b_{e}
$$

where $L^{\dagger}$ is the Laplacian pseudoinverse, and $b_{e}$ is the signed incidence (column) vector associated with edge $e$, with nonzero entries for the two nodes adjacent to that edge:

$$
\left(b_{e}\right)_{i}=\left\{\begin{array}{rl}
+1 & i=v_{1} \\
-1 & i=v_{2} \\
0 & \text { otherwise }
\end{array}\right.
$$

Aside from its algorithmic applications, $\Omega$ has a variety of intuitive interpretations. For example, it is: the fraction of spanning trees that include this edge (Bollobás 2001); the fraction of random walkers that use this edge during their stochastic transit between the two nodes joined by this edge (Tetali 1991); and, if one imagines the network as an electrical circuit where all the edges have unit resistance, it is the voltage difference between the two nodes when passing a unit of current between them (Christiano et al. 2011; Chandra et al. 1989).

Hence, an edge with higher effective resistance is more important for diffusion between its adjacent nodes. In the context of authorship networks such as ours, these connections 
often form "bridges" between communities, whereas edges that are more redundant (i.e., have lower effective resistance) will tend to appear within well-connected groups.

\section{Contraction importance $\Psi$}

However, the effective resistance measure assigns its maximal value $(\Omega=1)$ to every edge whose removal would lead to a disconnection of the network, regardless of the sizes of the resulting components. This applies not only to edges that would disconnect large groups, but also to isolated edges and edges connecting a single node at the periphery of the network, which are intuitively less important. Hence, we also consider the "contraction importance" $\Psi$ of edges, a recently proposed measure that is sensitive to these differences and is also derived from the graph Laplacian (Bravo-Hermsdorff and Gunderson 2019).

The contraction importance reflects how much the dynamics of diffusion throughout the network would change if an author were merged with their publication. Specifically, it is the change in the Laplacian pseudoinverse incurred by this merge, as quantified by the Frobenius norm. With this measure, an author who is a bridge between two communities is considered more important than an author who is only connected to the periphery of the network (see Fig. 5). The contraction importance $\Psi$ of an edge $e$ is defined as:

$$
\Psi_{e}=\frac{b_{e}^{\top} L^{\dagger} L^{\dagger} b_{e}}{b_{e}^{\top} L^{\dagger} b_{e}}
$$

To summarize, while both $\Omega$ and $\Psi$ give a measure of the importance of an edge to the overall connectivity of a network, there are some notable differences, particularly in the way they treat edges whose removal would disconnect a component of the network. The quantity $\Omega$ considers how much the diffusion between a given author and one of their publications would be reduced if this connection were to be deleted (i.e., removing the author from this publication). Thus, if this connection is the only path from this author to this publication, it would be considered maximally important $(\Omega=1)$. In contrast, $\Psi$
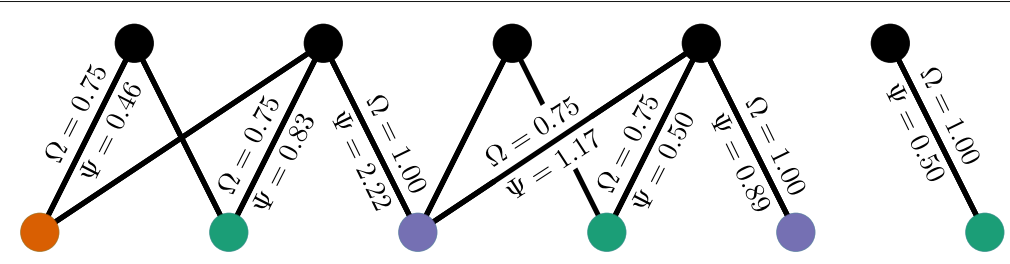

Fig. 5 Schematic illustrating the difference between effective resistance $\Omega$ and contraction importance $\Psi$ in a gendered bipartite authorship network. This example network has the basic structure of the bipartite authorship network we studied: a collection of publications (represented by the black nodes) connected to their authors (represented by the nodes with colors corresponding to their assigned gender labels: "women", "men" and "unknown"). Both effective resistance and contraction importance quantify the importance of an edge to diffusion in the network, albeit in different ways. The effective resistance $\Omega$ takes one of two values throughout the entire example network: either 1.00 (if its removal would disconnect nodes joined by that edge) or 0.75 (if the edge participates in a $K_{2,2}$ substructure). In contrast, the contraction importance $\Psi$ is sensitive to the edge position relative to the rest of the network; an edge whose removal splits a network into two components with $n_{A}$ and $n_{B}$ nodes has importance $\Psi=n_{A} n_{B} / n_{A \cup B}$. For example, an isolated edge has $\Psi=0.50$ and an edge that connects to the giant component via one node has $\Psi \rightarrow 1$. In addition, edges that are more integral to the horizontal diffusion are given a higher $\Psi$. For instance, compare the edge near the center with $\Psi=1.17$ and the edge to its right with $\Psi=0.50$; the former is clearly more important for communicating between the left and right portions of the network, despite the fact that they both have $\Omega=0.75$ 
measures how much the contraction of an author-publication connection (i.e., considering this author and publication as the same entity) would alter diffusion throughout the entire network. Therefore, contraction importance does not treat all edges with $\Omega=1$ equally, instead assigning smaller values to those that are less important for large-scale diffusion throughout the network (indeed the case for most edges with $\Omega=1$ in the INFORMS network).

Effective resistance $\Omega$ and contraction importance $\Psi$ distributions in the INFORMS network When comparing the relative importance of different edges, one should consider their ratio. Hence, we take the log of these measures before computing means and other statistics. Fig. 6 displays the histogram of both measures in the entire cumulative INFORMS network, illustrating how they provide relevant information about the connectivity of the network. For example, the contraction importance exhibits peaks at $n \in \mathbb{N}$, corresponding to edges whose removal would separate $n$ nodes from the bulk of the network, and the effective resistance exhibits peaks at $3 / 4$ and $2 / 3$, corresponding to the complete bipartite subgraphs $K_{2,2}$ and $K_{2,3}$ (i.e., two authors/publications connected to two or three publications/authors).

Both measures have similarly shaped distributions when conditioned on the genders. However, the average log contraction importance of authorships by women is lower than that for authorships by men, while the average log effective resistance of authorships by women is higher. In order to provide a meaningful comparison of these measures between the genders, we constructed a null model that explicitly replicates the yearly degree distributions for publications, women authors, men authors and unknown authors, but assigns author-publication connections irrespective of gender.

\section{A degree-preserving temporal and geometric null model with emergent communities}

Gender differences in measures of authorship structural importance could be attributable to gender differences in local statistics. For example, edges connecting nodes with higher degrees tend to have lower effective resistance, thus the sole fact that men have a higher number of publications could directly lead to the observed difference between the effective resistance of authorships by women and men. Therefore, in order to determine if

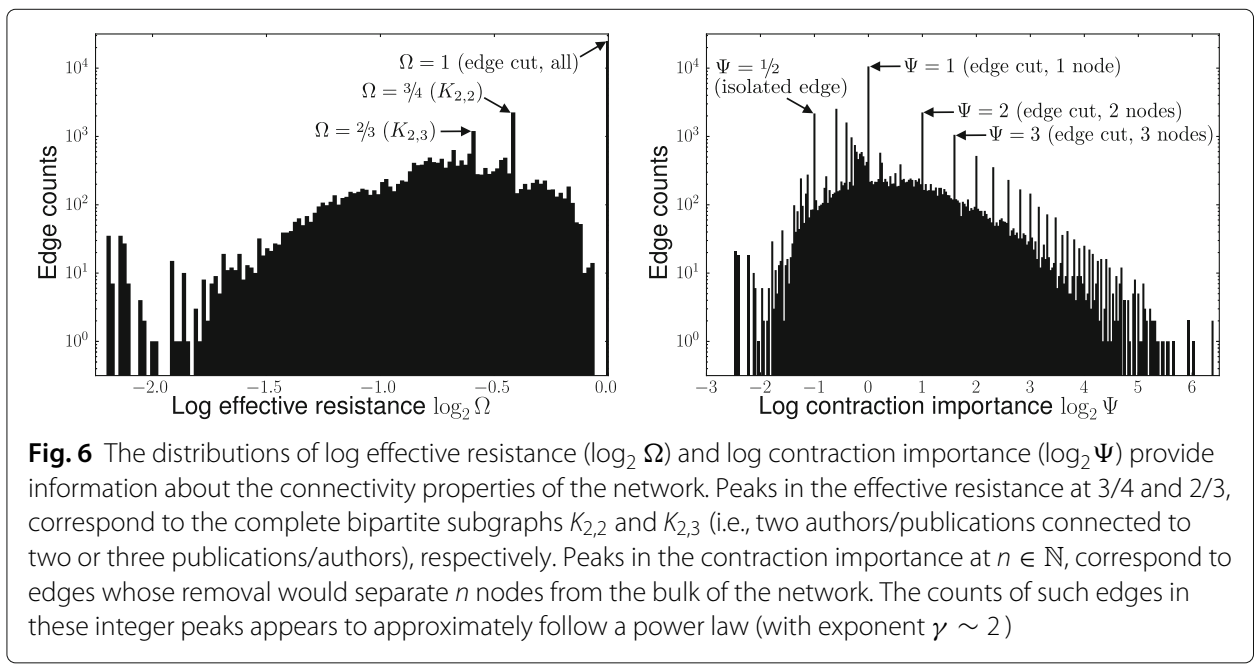


differences in collaboration patterns require an explanation beyond basic local statistics, it is crucial to have a null model that replicates the relevant local properties of the original network. Such a null model can then be used to determine whether other properties of interest are simply consequences of these local statistics.

In this section, we describe a novel degree-preserving temporal and geometric null model with emergent communities, which we believe could be of independent interest. The model employs a combination of geometric embedding to mimic clustering due to node similarity (Krioukov 2016), and a self-reinforcing node-placement mechanism to encourage community formation (Zuev et al. 2015).

The model explicitly replicates the observed yearly degree distributions for publications, as well as the gender and publication histories of each individual author (i.e., their number of publications each year). These are taken from the data as fixed inputs to the model. As we are interested in the effect of gender on collaboration patterns, our null model is blind to gender when assigning author-publication connections.

Aside from these inputs, the model has two free parameters: $D$, the dimension of the embedding space, and $n_{\text {nei }}$, a parameter controlling the propensity for clustering. We set these parameters so as to best match the data in other relevant aspects, such as the component size distribution over time.

Specifically, for each year, the model performs the following steps:

1. Choose a length scale $\lambda$ for this year, such that there will be $n_{\text {nei }}$ expected number of authors within a ball of radius $\lambda$, i.e., $\lambda=\left(\frac{n_{\text {nei }}}{n_{\text {tot }}} \frac{\Gamma(D / 2+1)}{\pi^{D / 2}}\right)^{1 / D}$, where $n_{\text {tot }}$ is the number of author nodes already placed (from previous years).

2. Add the new author nodes from this year (simultaneously) at locations $x_{a}$ in a $D$-dimensional unit torus, with probability proportional to the "attractiveness" of that location:

$$
p\left(x_{a}\right) \propto \sum_{i=1}^{n_{\text {tot }}} \exp \left(-\frac{\left|x_{a}-x_{i}\right|^{2}}{2 \lambda^{2}}\right),
$$

where $x_{i}$ are the locations of the existing $n_{\text {tot }}$ author nodes from previous years.

3. Add a "half-edge" to each of the author nodes, for each publication they authored this year.

4. Sequentially add the publication nodes from this year, in order of decreasing number of authors. The probability of placing a publication at a location $x_{p}$ is

$$
p\left(x_{p}\right) \propto \prod_{i=1}^{d_{p}} \exp \left(-\frac{\left|x_{p}-x_{i}\right|^{2}}{2 \lambda^{2}}\right),
$$

where $d_{p}$ is the number of authors on the publication, and $x_{i}$ are the locations of the nearest $d_{p}$ authors with available half-edges.

5. The publication then connects to each of its requisite number of authors with probability

$$
p\left(x_{p} \leftrightarrow x_{a}\right) \propto \exp \left(-\frac{\left|x_{p}-x_{a}\right|^{2}}{2 \lambda^{2}}\right),
$$

where $x_{p}$ and $x_{a}$ are the positions of the publication and an author with an unused half-edge, respectively.

6. Repeat steps 4-5 until all publications haven placed (and author half-edges used). 
In the network created by this algorithm, every woman and man author has exactly the same number of publications for each year as they do in the INFORMS network. However, importantly, the mechanism by which the null model decides which publication to give to an author does not consider gender, and thus the resulting networks can be used as a null model to study gender differences in global statistics.

\section{The null model mimics emergent network properties}

Aside from the explicitly matched temporal degree distributions, our null model is able to capture other relevant network features (see Fig. 7). Namely, certain settings of the free parameters (e.g., $D \gtrsim 4$ ) allow the null model to replicate the initial appearance of the giant component, while others (e.g., $D \lesssim 4$ ) match better the behavior in later years.

\section{Correlations between author gender}

\section{and the role of their authorships in the global network structure}

We used our null model to evaluate the effect of gender in determining the importance of an authorship to the global network structure. For each year, we calculated the difference in average edge importance measures $\left(\log _{2} \Omega\right.$ and $\left.\log _{2} \Psi\right)$ between the genders for the INFORMS network and for multiple simulations of the null model (all with $D=4$ and $n_{\text {nei }}=1$ ). As shown in Fig. 8, the data and null model deviate considerably.

Until around 1980, the null model predicted no difference between the effective resistance $\Omega$ of authorships by women and men. This is likely due to the prevalence of author-publication connections with $\Omega=1$. In fact, until 1966, there were only 21 women in the dataset, and all had only one publication: this means that all of their effective resistances are precisely 1 in both the data and the null model. Moreover, as the null model predicted no difference during this period between the effective resistance of authorships by women and men, authorships by men similarly have effective resistance close to 1 . This is in contrast to the easily-discernible clustering present in the earlier years of the actual INFORMS network - our choice of parameters for the null model resulted in networks containing more tree-like components (for which all edges have $\Omega=1$ ) during this period. We chose these parameters in order to qualitatively match the bulk of the available data, namely the emergence $(\sim 1980)$ and size of the giant component. While our model captured this feature of the data, none of the parameter settings we tried allowed it to capture the clustering present before $\sim 1980$. Therefore, it is difficult to interpret

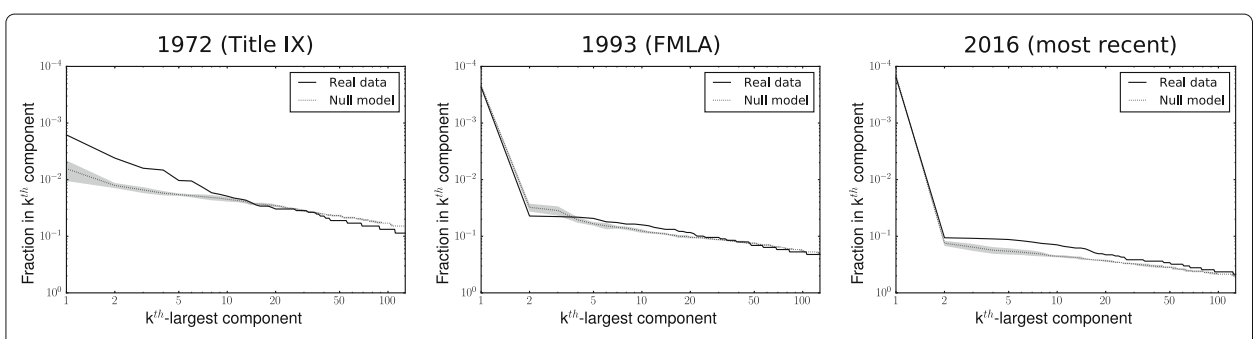

Fig. 7 The null model qualitatively mimics the emergence and size of the giant component. Plots show the fraction of authors in each of the 128 largest components in the cumulative network up to the indicated year. Solid black curve denotes the INFORMS network, and the shading denotes the range ( \pm 1 standard deviation) of a typical run of the null model (with parameters $D=4$ and $n_{\text {nei }}=1$ ) about the mean of 8 instances of the null model (dotted curve) 
differences between the null model and the actual data during this early period. The average contraction importance does, however, show gender differences in the null model during this period (Fig. 8, right), highlighting the higher sensitivity of this measure of edge importance.

From around 1980 until around 2005, the null model begins to predict gender differences in both $\Omega$ and $\Psi$. This is a particular relevant period as $\sim 1980$ coincides with the onset of a consistent increase in women participating in INFORMS and the emergence of the giant component (see Fig. 1 and Additional file 1: Video S1). Moreover, we chose the parameters of the null model to best replicate the component size distribution during this period (see Fig. 7). Interestingly, the two measures deviate in opposite directions: the null model predicts that women should have a higher effective resistance and lower contraction importance than men. Neither of these changes were observed in the actual data, which instead display gender differences closer to zero for both $\Omega$ and $\Psi$. This suggests that the gender differences in global statistics found in the INFORMS network during this period cannot be explained by only local statistics. Finally, from $\sim 2005$, the predicted gender differences are closer to those observed in the actual data, where authorships by women have effective resistance slightly higher and contraction importance slightly lower than authorships by men. This suggests that the more recent gender differences in global statistics can be explained mostly by differences in local statistics, such as the underrepresentation of women in the network.

\section{Discussion}

In this work, we investigated the correlation between gender and scientific publication patterns by analyzing a temporal bipartite network between authors and publications in the INFORMS journals. Our study provides two methodological contributions: 1) We develop a simple temporal geometric null model (with two free parameters) that encourages emergent communities (a hallmark feature of real-world networks); and 2) We present a case study for applying effective resistance and contraction importance (two measures of edge importance related to diffusion throughout a network) to quantify the importance of different connections to the global structure of a network.
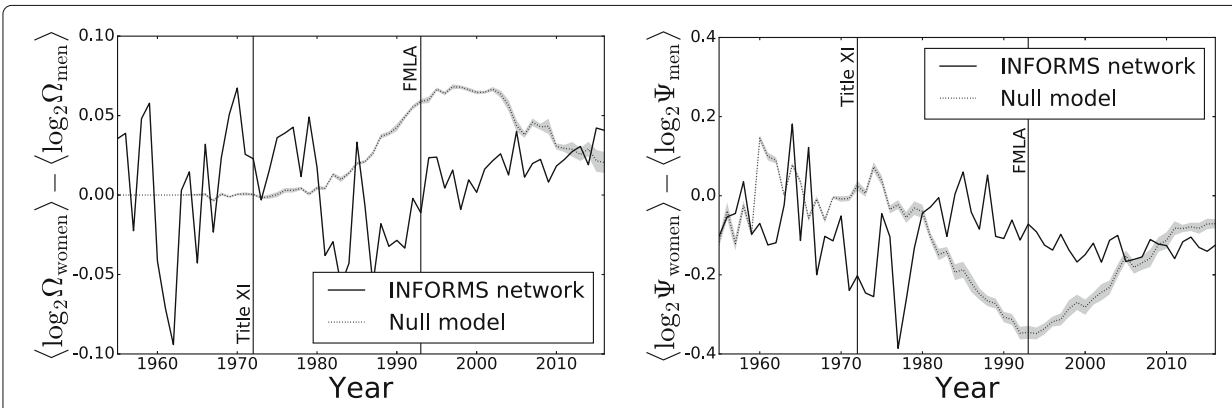

Fig. 8 Differences in measures of authorship importance of women and men have recently tapered off. Gender differences (women minus men) over time in average edge importance measures (effective resistance, $\log _{2} \Omega$, left; and contraction importance, $\log _{2} \Psi$, right). The solid curve is the difference in the INFORMS dataset, and the dotted curve in the null model, which replicates the yearly degree distributions for publications, women authors, and men authors, but assigns author-publication connections irrespective of gender. The shading denotes the range ( \pm 1 standard deviation) of a typical run of the null model (with parameters $D=4$ and $n_{\text {nei }}=1$ ) about the mean of 8 instances of the null model (dotted curve) 
While conventional measures of importance/centrality (e.g., edge betweenness (Girvan and Newman 2002)) may give qualitatively similar results, the measures we chose are particularly relevant due to their use in the analysis and implementation of a variety of graph algorithms. For example, they serve as a measure of edge importance in several graph reduction algorithms (Spielman and Srivastava 2011; Fung et al. 2019), which are often used as primitives in other efficient algorithms for massive networks. As the field of applied network science often deals with such structures, it is relevant to investigate how such algorithms might interact with datasets containing metadata (such as node labels).

At the level of local statistics, we found that both the fractions of new women authors and of authorships by women in the INFORMS network have been increasing steadily since $\sim 1980$ (see Fig. 1). Before then, the fraction of new women authors hovered around less than $3 \%$. While multiple factors may have contributed to this sudden change in derivative, it is not unreasonable to hypothesize that the introduction of Title IX legislation in the United States in 1972 played a role that is still unfolding, and that such quantification of long-term effects could serve as a good argument for similar policies.

Despite the continued increase in the fraction of new women authors in INFORMS, more than four decades after Title IX, women still comprise less than $25 \%$ of the network (Fig. 1). Moreover, women are disproportionately underrepresented among authors with many publications (Figs. 2 and 3). While this could be due to a variety of factors, we draw attention to gender biases in the peer-review process and visibility of researchers in the community (e.g., invitations to present work at conferences and colloquia (Nature 2016)). These issues have been the focus of several recent studies. For example, Murray et al. (2018) studied the review process in the journal eLife, and found that reviewers appear to favor authors with demographic characteristics (gender and nationality) similar to their own. In analyzing data from a longitudinal experiment by the Canadian Institutes of Health Research, Witteman et al. (2019) found that women were less likely to be awarded a grant when the review focus was on "the principal investigator" as compared to "the proposed science". Nittrouer et al. (2017) found that men were more likely than women to be colloquium speakers at top US universities (even when controlling for speaker rank and the gender ratio in the field, and despite men and women declining invitations at similar rates). Due to enhanced scrutiny and attention to these issues, these trends have been changing more recently. For example, BiasWatchNeuro.com (BiasWatchNeuro 2019), a website dedicated to monitoring gender representation of invited speakers at conferences in the field of neuroscience, has documented a steady increase in the rate of women invited to present at conferences, with this rate now approaching a conservative estimate of the base rate of women in the field ( $30 \%)$.

Indeed, much effort is being invested in methods for mitigating these biases, such as compiling online lists of women researchers to facilitate their invitation as conference speakers and nomination for prizes (Request a Woman Scientist 2019; AcademiaNet 2019; Women in Neuroscience Repository 2019); making data on the gender balance in conferences and panels more visible online; and encouraging journals to adopt a policy of double-blind reviews, which has been showed to reduce biases (such as increasing representation of women authors (Budden et al. 2008)). Such efforts might help improve scientific productivity by increasing gender heterogeneity in the scientific workforce (Campbell et al. 2013). For a thorough review on the issue of gender bias in science (with a focus on neuroscience) and recommended efforts to mitigate it, see Schrouff et al. (2019). 
By accurately quantifying the nature of systematic asymmetries, one can more precisely inform relevant policies (Fagan et al. 2018; Luke et al. 2016; Okamoto 2014). For example, simulations of STEM faculty retention in a US university (Thomas et al. 2015) suggest that in order for gender parity to be reached in those data, the higher rate of women resigning must be addressed. A recent study (Cech and Blair-Loy 2019) analyzing a longitudinal national survey of US STEM professionals, suggests that this higher rate might be due to women leaving their full-time STEM jobs upon becoming parents for the first time $(\sim 43 \%$ resignation of women vs. $\sim 23 \%$ of men). Additionally, it was found that, among those that continued to hold full-time jobs, parents were less likely to remain in their STEM jobs than their child-less peers. This suggests that policies to render work in STEM fields more compatible with caregiving may be critical to increase gender diversity. Likewise, network science analysis such as ours could help inform diversity efforts by indicating potential connections that are more likely to decrease gender disparities, for example, by funding research with author-publication connections that are diverse and important to the overall connectivity of the network. This type of analysis could also help evaluate the global impact of such efforts. For instance, future work could focus on trends that might correlate with more recent diversity initiatives in the INFORMS society (e.g., in 2006 and 2017). However, as the time-scale of our dataset is on the order of a typical career length, results during the last decade or so are still unfolding; thus, it is presently difficult to measure the effect of policy changes during this time.

To shed light on where to focus such efforts, future work could focus on correlations between gender differences and various subcommunities. For example, in the INFORMS dataset, some journals have a relatively higher rate of women authors (e.g., $\sim 28 \%$ in Organization Science), while others have a much lower rate (e.g., $\sim 8 \%$ in Mathematics of Operations Research; see Additional file 3: Figure S1). Another interesting research avenue is to understand how particular collaboration patterns and homophily correlate with academic success (as measured, for instance, by number of future publications). The period of 1980-2005 in the INFORMS network is especially interesting in this regard, given that, on average, women and men had more similar effective resistance and contraction importance in those years than predicted by the local statistics incorporated in our null model. This discrepancy between the data and the null model could indicate that women entering the INFORMS network had similar network roles as men. Another possibility is that women and men formed somewhat separate collaborative networks with similar statistics. Future work could investigate whether these, or other patterns, are dominant in the network.

It is important to recognize the limitations of our methodology. For instance, our data cleaning methods are likely biased; Genderize.io (the software we used to classify the genders of the authors) has a higher misclassification rate for non-Western names (Santamaría and Mihaljević 2018). Moreover, as first names in many contemporary Western cultures are frequently indicative of only two genders, the API reduces the multidimensional continuum of genders to a single binary variable. In addition, the data selected for manual gender classification (i.e., all authors with more than six publications) are also likely correlated with gender, ${ }^{3}$ biasing the ratio of known author genders. It is also 
important to acknowledge that our simple and physically-motivated null model does not replicate all of the relevant gender-ambivalent network properties. For example, we did not precisely match the component size distributions throughout the development of the network; lower dimensional embeddings better matched the earlier distributions, and higher dimensional embeddings better matched the later distributions. A time-varying $n_{\text {nei }}$ will likely describe the data better, and thus provide a better comparison for evaluating the roles of women and men in the network over time, in particular, in the early years. However, from a practical point of view, those early years may be less informative regarding the effectiveness of current policies for mitigating gender biases.

Overall, our results indicate that the INFORMS society remains far from gender parity in many important local statistics. However, since $\sim 1980$ these differences have been steadily decreasing, and recent $(\gtrsim 2005)$ global statistics are more in agreement with our null model, indicating progress. More generally, we hope that building a quantitative understanding of gender publication and collaboration patterns in academia will ultimately help accelerate the path towards gender equality by bringing awareness to the issue and informing future studies and policies.

\section{Supplementary information}

Supplementary information accompanies this paper at https://doi.org/10.1007/s41109-019-0214-4.

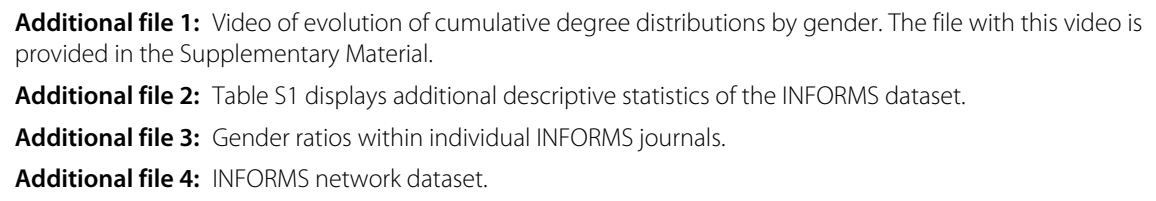

Abbreviations

INFORMS: Institute for operations research and the management sciences; STEM: Science, technology, engineering, and mathematics; ORSA: The operations research society of America; TIMS: The institute of management sciences; Title IX: Title IX of the Education Amendments Act of 1972; FMLA: The Family and Medical Leave Act of 1993

\section{Authors' contributions}

$E R$, JM, and MEH conceived the overarching question and constructed the dataset. ER, GBH, MEH and VF performed data cleaning and validation. GBH and LMG designed and performed the analysis and discussed the results with ER, MEH, VF and $Y N$. GBH, LMG, VF, and YN wrote the manuscript with input from ER and MEH. All authors read and approved the final manuscript.

Funding

This work was funded in part by a diversity grant from the Simons Foundation (VF,YN) and internal funds from Princeton University $(\mathrm{GBH}, \mathrm{YN})$.

\section{Availability of data and materials}

The dataset acquired and analyzed for this publication is available in the supplementary information (Additional file 4) as well as at https://github.com/Gecia/INFORMS_TemporalGenderedAuthorshipNetwork.

\section{Competing interests}

The authors declare that they have no competing interests.

\section{Author details}

${ }^{1}$ Princeton Neuroscience Institute, Princeton University, Washington Rd, 08544, Princeton, NJ, USA. ${ }^{2}$ Department of Psychology, Princeton University, Washington Rd, 08544, Princeton, NJ, USA. ${ }^{3}$ Product Data Science, Grubhub Inc., 1065 6th Ave, 10018, New York, NJ, USA. ${ }^{4}$ Department of Data Science, IBM T.J. Watson Research Center, 1101 Kitchawan Rd, 10598 Yorktown Heights, NJ, USA. 5 Princeton Plasma Physics Laboratory, Princeton University, 100 Stellarator Rd, 08540 Princeton, NJ, USA. ${ }^{6}$ Lerner Center for Public Health Promotion (Center for Policy Research), Maxwell School of Citizenship and Public Affairs, Syracuse University, 426 Eggers Hall, 13244 Syracuse, NJ, USA. 


\section{References}

Abbasi A, Chung KSK, Hossain L (2012) Egocentric analysis of co-authorship network structure, position and performance. Inf Proc Manag 48(4):671-679. https://doi.org/10.1016/j.ipm.2011.09.001

About INFORMS (2019). https://www.informs.org/About-INFORMS. Accessed 19 Oct 2019

AcademiaNet (2019) The Portal to Excellent Women Academics. http://www.academia-net.org/. Accessed 19 Oct 2019

Araújo EB, Araújo NAM, Moreira AA, Herrmann HJ, Andrade JS (2017) Gender differences in scientific collaborations: Women are more egalitarian than men. PLOS ONE 12(5):0176791. https://doi.org/10.1371/journal.pone.0176791

Bollobás B (2001) Random Graphs. Cambridge University Press, Cambridge New York

Bravo-Hermsdorff G, Gunderson LM (2019) A unifying framework for spectrum-preserving graph sparsification and coarsening. Neural Inf Proc Syst (NeurlPS). http://arxiv.org/abs/1902.09702

Budden AE, Tregenza T, Aarssen LW, Koricheva J, Leimu R, Lortie CJ (2008) Double-blind review favours increased representation of female authors. Trends Ecol Evol 23(1):4-6. https://doi.org/10.1016/j.tree.2007.07.008

BiasWatchNeuro (2019). https://biaswatchneuro.com/. Accessed 19 Oct 2019

Campbell LG, Mehtani S, Dozier ME, Rinehart J (2013) Gender-heterogeneous working groups produce higher quality science. PloS one 8(10):79147. https://doi.org/10.1371/journal.pone.0079147

Cech EA, Blair-Loy M (2019) The changing career trajectories of new parents in STEM. Proc Nat Acad Sci:201810862. https://doi.org/10.1073/pnas.1810862116

Chandra AK, Raghavan P, Ruzzo WL, Smolensky R (1989) The electrical resistance of a graph captures its commute and cover times. In: Proceedings of the Twenty-first Annual ACM Symposium on Theory of Computing. STOC '89. ACM, New York. pp 574-586. https://doi.org/10.1145/73007.73062

Chen Y, Ding C, Hu J, Chen R, Hui P, Fu X (2017) Building and analyzing a global co-authorship network using google scholar data. In: Proceedings of the 26th International Conference on World Wide Web Companion. WWW '17 Companion. International World Wide Web Conferences Steering Committee, Republic and Canton of Geneva, Switzerland. pp 1219-1224. https://doi.org/10.1145/3041021.3053056

CrossRef REST API (2019). http://www.crossref.org/services/metadata-delivery/rest-api/

Chouldechova A, Roth A (2018) The frontiers of fairness in machine learning. arXiv preprint arXiv:1810.08810. https://arxiv. org/abs/1810.08810

Christiano P, Kelner JA, Madry A, Spielman DA, Teng S-H (2011) Electrical flows, Laplacian systems, and faster approximation of maximum flow in undirected graphs. In: Proceedings of the Forty-third Annual ACM Symposium on Theory of Computing. STOC '11. ACM, New York. pp 273-282. https://doi.org/10.1145/1993636.1993674

Denton B (2017) INFORMS membership - diversity, equity and inclusion. OR/MS Today 44(6):8-9

Family and Medical Leave Act of 199329 U.S. Code Chapter 28. J Res Adm 49:76-99

Fagan J, Eddens K, Dolly J, Vanderford N, Weiss H, Levens J (2018) Assessing research collaboration through co-authorship network analysis

Fiedler M (1973) Algebraic connectivity of graphs. Czechoslovak Math J 23:298-305

Fung W-S, Hariharan R, Harvey NJ, Panigrahi D (2019) A general framework for graph sparsification. SIAM J Comput 48(4):1196-1223. https://doi.org/10.1137/16M1091666

Genderize.io (2019). http://www.genderize.io

Girvan M, Newman MEJ (2002) Community structure in social and biological networks. Proc Nat Acad Sci 99(12):7821-7826. https://doi.org/10.1073/pnas.122653799

Hall JR (1983) An issue-oriented history of TIMS. Interfaces 13(4):9-19

Helmer M, Schottdorf M, Neef A, Battaglia D (2017) Gender bias in scholarly peer review. eLife 6. https://doi.org/10.7554/ elife. 21718

Horner P (2017) History lesson: The evolution of INFORMS. OR/MS Today 44(1)

INFORMS Publications (2019). https://www.informs.org/Publications

Jadidi M, Karimi F, Lietz H, Wagner C (2018) Gender disparities in science? Dropout, productivity, collaborations and success of male and female computer scientists. Adv Compl Syst 21(03n04):1-23. https://doi.org/10.1142/ S0219525917500114

Karimi F, Mayr P, Momeni F (2018) Analyzing the network structure and gender differences among the members of the Networked Knowledge Organization Systems (NKOS) community. Int J Digital Libraries. https://doi.org/10.1007/ s00799-018-0243-0

Kitsak M (2017) Latent geometry of bipartite networks. Phys Rev E 95(3). https://doi.org/10.1103/PhysRevE.95.032309

Klerman JA, Daley K, Pozniak A (2012) Family and medical leave in 2012. Tech Rep

Krioukov D (2016) Clustering implies geometry in networks. Phys Rev Lett 116(20). https://doi.org/10.1103/PhysRevLett. 116.208302

Leslie S-J, Cimpian A, Meyer M, Freeland E (2015) Expectations of brilliance underlie gender distributions across academic disciplines. Science 347(6219):262-265. https://doi.org/10.1126/science.1261375. http://arxiv.org/abs/http://science. sciencemag.org/content/347/6219/262.full.pdf

Liu X, Bollen J, Nelson ML, de Sompel HV (2005) Co-authorship networks in the digital library research community. Inf Proc Manag 41(6):1462-1480. https://doi.org/10.1016/j.ipm.2005.03.012

Liu LT, Dean S, Rolf E, Simchowitz M, Hardt M (2018) Delayed impact of fair machine learning. In: Dy J, Krause A (eds). Proceedings of the 35th International Conference on Machine Learnin. Proceedings of Machine Learning Research. PMLR, Stockholmsmässan, Stockholm Sweden Vol. 80. pp 3150-3158. http://proceedings.mlr.press/v80/liu18c.html

Luke DA, Baumann AA, Carothers BJ, Landsverk J, Proctor EK (2016) Forging a link between mentoring and collaboration: a new training model for implementation science. Implement Sci 11(1):137. https://doi.org/10.1186/s13012-016-0499-y

Moss-Racusin CA, Dovidio JF, Brescoll VL, Graham MJ, Handelsman J (2012) Science faculty's subtle gender biases favor male students. Proc Nat Acad Sci 109(41):16474-16479. https://doi.org/10.1073/pnas.1211286109. http://arxiv.org/ abs/https://www.pnas.org/content/109/41/16474.full.pdf

Murray D, Siler K, Lariviére V, Chan WM, Collings AM, Raymond J, Sugimoto CR (2018) Gender and international diversity improves equity in peer review. bioRxiv. https://doi.org/10.1101/400515. http://arxiv.org/abs/https://www.biorxiv. org/content/early/2018/08/29/400515.full.pdf 
Nagurney A (2007) What Professional Societies Can Do from the Top Down. Presented at Women in Science Panel of the Association for Women In Science Massachusetts Chapter. https://supernet.isenberg.umass.edu/visuals/nagurneyawis.pdf

Nature (2016) Women need to be seen and heard at conferences 538(7625):290-290. https://doi.org/10.1038/538290b

Nittrouer CL, Hebl MR, Ashburn-Nardo L, Trump-Steele RCE, Lane DM, Valian V (2017) Gender disparities in colloquium speakers at top universities. Proc Nat Acad Sci 115(1):104-108. https://doi.org/10.1073/pnas.1708414115

Okamoto J (2014) Scientific collaboration and team science: a social network analysis of the centers for population health and health disparities. Transl Behavior Med 5(1):12-23. https://doi.org/10.1007/s13142-014-0280-1

ORSA/TIMS collaboration (1973) INFORMS J Appl Anal 3(4):32-42. https://doi.org/10.1287/inte.3.4.32

Public Law No. 92 - 318, 86 Stat. 235 (June 23, 1972), codified at 20 U.S.C. $\$ \$ 1681-1688$

Raymond J (2013) Most of us are biased. Nature 495(7439):33-34. https://doi.org/10.1038/495033a

Request a Woman Scientist (2019). https://500womenscientists.org/request-a-scientist

Santamaría L, Mihaljević H (2018) Comparison and benchmark of name-to-gender inference services. PeerJ Comput Sci 4:156. https://peerj.com/articles/cs-156/

Schrouff J, Pischedda D, Genon S, Fryns G, Pinho AL, Vassena E, Liuzzi AG, Ferreira FS (2019) Gender bias in (neuro) science: facts, consequences and solutions. Eur J Neurosci. https://doi.org/10.1111/ejn.14397

Spielman DA, Srivastava N (2011) Graph sparsification by effective resistances. SIAM J Comput 40(6):1913-1926. https:// doi.org/10.1137/080734029

Teng S-H (2010) Theory and Applications of Models of Computation. In: Kratochvíl J, Li A, Fiala J, Kolman P (eds). Springer, Berlin, Heidelberg. pp 2-14

Tetali P (1991) Random walks and the effective resistance of networks. J Theoret Prob 4:101-109. https://doi.org/10.1007/ BF01046996

The Institute for Operations Research and the Management Sciences (2019) INFORMS PubsOnLine. http://www. pubsonline.informs.org

Thomas NR, Poole DJ, Herbers JM (2015) Gender in science and engineering faculties: Demographic inertia revisited. PLOS ONE 10(10):1-9. https://doi.org/10.1371/journal.pone.0139767

US Department of Justice (2012) Equal Access to Education: Forty Years of Title IX. https://www.justice.gov/sites/default/ files/crt/legacy/2012/06/20/titleixreport.pdf

Walters J, McNeely CL (2010) Recasting Title IX: Addressing gender equity in the science, technology, engineering, and mathematics professoriate. Rev Policy Res 27(3):317-332. https://doi.org/10.1111/j.1541-1338.2010.00444.x

West JD, Jacquet J, King MM, Correll SJ, Bergstrom CT (2013) The role of gender in scholarly authorship. PLoS ONE 8(7):66212. https://doi.org/10.1371/journal.pone.0066212

Women in Neuroscience Repository (2019). https://www.winrepo.org/

Witteman HO, Hendricks M, Straus S, Tannenbaum C (2019) Are gender gaps due to evaluations of the applicant or the science?A natural experiment at a national funding agency. The Lancet 393(10171):531-540. doi:https://doi.org/10. 1016/S0140-6736(18)32611-4

Zuev K, Boguñá M, Bianconi G, Krioukov D (2015) Emergence of soft communities from geometric preferential attachment. Sci Rep 5(1). https://doi.org/10.1038/srep09421

\section{Publisher's Note}

Springer Nature remains neutral with regard to jurisdictional claims in published maps and institutional affiliations.

\section{Submit your manuscript to a SpringerOpen ${ }^{\circ}$ journal and benefit from:}

- Convenient online submission

- Rigorous peer review

- Open access: articles freely available online

- High visibility within the field

- Retaining the copyright to your article

Submit your next manuscript at $\boldsymbol{\triangleright}$ springeropen.com 